\title{
Cell Proliferation and Epithelia Profile in Prostate Cancer and Benign Prostatic Hyperplasia
}

\author{
Ogundele O.M. ${ }^{1,3}$, Adegun P.T. ${ }^{*}$, Falode D.T. ${ }^{4}$, Agbaje A.M. ${ }^{6}$, Ajonijebu D.C. ${ }^{3}$, Omoaghe A.O. ${ }^{3}$ and \\ Laoye, B.J. ${ }^{5}$ \\ ${ }^{I}$ Department of Anatomy, College of Medicine and Health Sciences, Afe Babalola University, Ado-Ekiti, Nigeria \\ ${ }^{2}$ Department of Urology and Oncology, University Teaching Hospital, Ado-Ekiti, Nigeria \\ ${ }^{3}$ Department of Physiology, Afe Babalola University, Ado-Ekiti, Nigeria \\ ${ }^{4}$ Department of Nursing Sciences, Afe Babalola University, Ado-Ekiti, Nigeria \\ ${ }^{5}$ Department of Human Biology, College of Sciences, Afe Babalola University, Ado-Ekiti, Nigeria \\ ${ }^{6}$ Department of Anatomy, College of Medicine, Ekiti State University, Ado-Ekiti, Nigeria
}

\begin{abstract}
Objectives: Prostate cancer (PCa) and Benign Prostatic Hyperplasia (BPH) are the leading cause of urinary tract disorders in males both of which are characterized by rapid proliferation of cells. This study characterizes both Ki-67 and E-Cadherin profiles in BPH and PCa biopsies to determine and compare the extent of aggressiveness of cell proliferation in $\mathrm{BPH}$ and $\mathrm{PCa}$.

Method: Biopsies were collected from patients clinically diagnosed to be suffering from PCa or BPH and were immunohistochemically labelled using Anti-Ki-67 and Anti-ECAD monoclonal antibodies. The sections were treated with urea followed by incubation in a microwave to activate the antigens. Primary antibody treatment was done in biotinylated goat serum, blocking was done with bovine serum albumin (BSA) and finally secondary antibody staining using Anti-Ki67 and Anti-ECAD monoclonal antibodies.

Results: The expression level of Ki-67 corresponded to the rate of proliferation and size of the tumor cell mass in both $\mathrm{PCa}$ and BPH. ECAD expression was highly positive in BPH and less positive in PCa- showing onset of malignancy in PCa. Certain cell clumps (metastases) stained highly positive to Ki-67 and were located at random intervals within the tissue of PCa biopsies, this type of distribution also coincided with ECAD immunonegativity.

Conclusion: Ki-67 expression indicates the rate of cell proliferation and the aggressiveness of such tumors, while ECAD shows defective cytoskeleton and extent of malignancy. Correlating ECAD and Ki-67 gives a direct relationship between the rate of cell division, tumor invasion and epithelia structure. This also covers both glandular and fibromuscular epithelium
\end{abstract}

Keywords: Ki-67, ECAD, Cell Proliferation, cancer, tumor, Prostate, BPH.

\section{INTRODUCTION}

Recent advances in immunopathogenesis of cancer have allowed comparative assessment of molecular biomarkers that are prognostic to identifying triad of pathological processes and developing prostatic disease conditions such as benign prostatic hyperplasia $(\boldsymbol{B P H})$ and prostate cancer (PCa) which presents similar clinical picture and pathogenic mechanisms- the triad comprises of assessment of PSA level, Digital rectal examination and Trans rectal ultrasound scan [1]. Epidemiological profile of prostate dysfunction shows that prostate cancer is the second leading cause of cancer

*Address correspondence to this author at the Department of Surgery, College of Medicine, Ekiti State University, Ado-ekiti, Nigeria;

Tel: +2348034531236; E-mail: patrickikelomo@yahoo.com related death in men and accounts for $15 \%$ and $4 \%$ cancer cases in developed and developing countries, respectively [2]. Whereas, more than two-thirds of men older than fifty years have histological evidence of $B P H$ whose proportion increases to $80 \%$ after age 70 [3].

Despite high prevalence of prostate cancer and $B P H$, optimal treatment remains a challenge for health providers worldwide. Management of these diseases have been widely reported to hinge on standard clinical factors including Gleason scoring system, PSA level and measures of tumor extent on biopsy and imaging [4]; these were considered inadequate. Hence, better and specific prognostic markers are required to determine the exact nature and origin of such a cancer - this will aid the determination of the molecular basis of such cancers and can be used in the prediction of its 
progression which is normally inaccurate using the existing clinical diagnostic methods. It is widely reported that the molecular basis of tumor aggressiveness is related to

1. Uncontrolled tumor proliferation activity - which can be assessed by PCNA and Ki-67 antigen;

2. Adhesion, migration and tumor cell invasiveness especially related to E-cadherin adhesion molecule;

3. Tumor angiogenesis - which can be immunochemically evaluated by CD34 expression [5, 6].

E-cadherin is a cell surface glycoprotein involved in calcium-dependent homotypic cell-to-cell adhesion. It is responsible for organization, maintenance and morphogenesis of epithelial tissues [7]. It is expressed in normal arachnoid tissue, epithelial tissue and in human meningioma cells. The cytoplasmic tail of the E-cadherin molecules bind to $\beta$-catenin to form the cadherin - actin filament complex, which is necessary for functional cell to cell adhesion [8,9]. Suppression of expression or cleavage of this adhesion molecule may result in cells acquiring a mesenchymal morphology and increased motility [10]. During cancer progression to an invasive state, intercellular adhesions between tumor cells are disrupted. Thus, aggressive tumor cells were hypothesized to have loss of $E$ cadherin. Also, decreased E-cadherin expression has repeatedly been shown to correlate with a loss of tumor differentiation and poor prognosis [6]. This is important in differentiating $\mathrm{PCa}$ from $\mathrm{BPH}$, although both are characterized by rapid cell proliferation, epithelia integrity might be retained in $\mathrm{BPH}$ while cancerous cells in $\mathrm{PCa}$ would normally detach from the epithelium as seen in low ECAD expression for this category. The extent of such ECAD decrease in $\mathrm{PCa}$ will also determine the relative rate of tumor cell detachment from the epithelium and otherwise the degree of aggressiveness of such tumors.

$\mathrm{Ki}-67$, a DNA binding protein, is a standard proliferation marker for predicting tumor development. It is expressed only in dividing cells, either normal or tumor, but absent in resting cells. Only cells that over express $p 53$ or $p 21$ may be assessed using Ki-67. Thus, the level of Ki-67 expression may be used to determine the cell proliferation status [11]. Typically, actively growing prostate cancer cells are few and the rapidity of growth is at a reduced rate. Hence, the greater the proportion of prostate tumor cells with $\mathrm{Ki}-67$ indicates the aggressive nature of the cancer. A study by Alan Pollack in 2003 [12], shows that when tumor cells stained for Ki-67 is greater than $7.1 \%$, it is an indication of significant increase risk of distant metastasis and death due to prostate cancer.

Although, recent studies have evaluated index of proliferation, apoptosis, hormone receptors and TGF- $\beta$ signaling using both animal and in-vitro models. Despite this, Ki-67, the most studied of any immunochemical biomarker in prostate cancer, remains an important prognostic factor in multivariate analysis. Combination with other predictive biomarkers, such as E-cadherin, may be beneficial for proper diagnosis and optimization of disease management and offset limitations of early immunochemical factors. This is believed to further help identify correlation between biomarkers and prognostic factors in cancer. This study, however, evacuates the distribution of Ki-67 and $E C A D$ in the epithelium and glandular tissue of clinically diagnosed $\mathrm{BPH}$ and $\mathrm{PCa}$ biopsies as a function of cell proliferation and metastasis to foster comparison and transition between $P C a$ and $B P H$.

\section{MATERIALS AND METHODS}

\section{Tissue Processing}

BPH and PCa samples (biopsies) were obtained from patients clinically diagnosed and histologically confirmed to have the condition(s). The biopsies were fixed in formolcalcium (4BPH and 4Pca) and processed histologically to obtain paraffin wax embedded sections at the pathology lab of University Teaching Hospital, AdoEkiti.

\section{Histology}

Tissue sections were processed for routine Hematoxylin and Eosin following the methods of Zhang et al. (2012) [13] to demonstrate the general morphology of the prostate tissue.

\section{IMMUNOHISTOCHEMISTRY}

Cell Proliferation Marker (Ki-67) and E-Cadherin (ECAD): the objective is to determine the expression of $\mathrm{Ki}$ 67 and ECAD in BPH and PCa prostate tissues and to evaluate the effects of these variations in the expression of these two proteins in the progression of BPH and PCA. Ki67 is also used in this study to determine progression of DNA damage in both conditions. The tissue biopsies were initially fixed in formolsaline and embedded in paraffin wax in preparation for antigen retrieval. The monoclonal antibodies [anti-Ki-67 and anti ECAD were procured from Standard Bio Techniques, China]. The reagents were purified according the quality assurance specifications and antibody was diluted to 1:500 in Phosphate buffer saline (PBS).

Procedure: the paraffin wax embedded sections were mounted on a glass slide in preparation for antigen retrieval where the slides were immersed in urea overnight and then placed in a microwave for 45 minutes to re-activate the antigens and proteins in the tissue sections. Primary antibody treatment involved treating the sections with biotinylated goat serum for one hour following which the sections were transferred to $1 \%$ bovine serum albumin (BSA) to block non-specific protein reactions. Secondary treatment involved the use of diluted anti-Ki-67 and anti-ECAD on the pretreated sections for one hour. The immunopositive reactions were developed using a polymer 3'3' Diaminobenzidine Tetrachloride (DAB) with colour intensification involving the use of mathenamine silver kit. The sections were counterstained in Hematoxylin and treated in $1 \%$ acid alcohol (freshly prepared).

\section{TRANSFORMATION}

Mathenamine silver intensification was used on the immunoperoxidase preparation after the peroxidase $/ \mathrm{H}_{2} \mathrm{O}_{2} / D A B$ reaction has been carried out to give a brown deposit. The sections were then counterstained in Hematoxylin. The counterstained sections were washed in running tap water, thoroughly rinsed in distilled water, and 


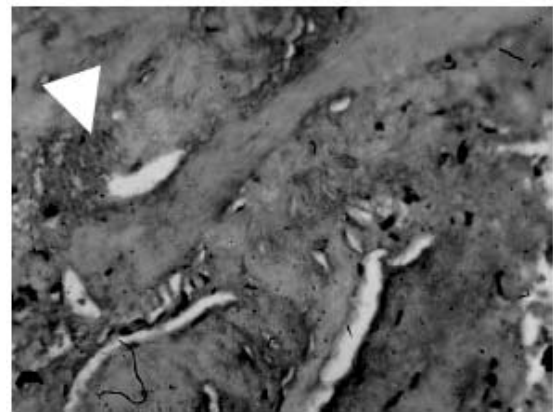

S09

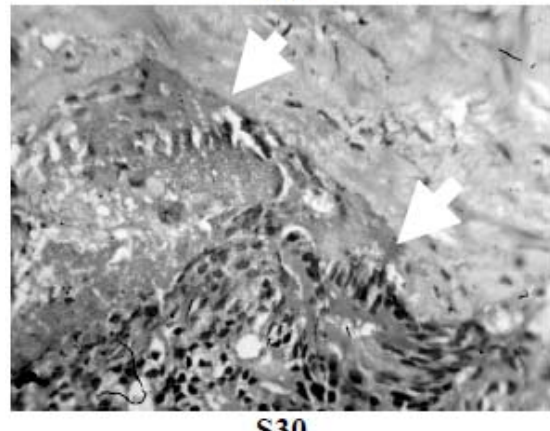

S30



S40 (BPH)

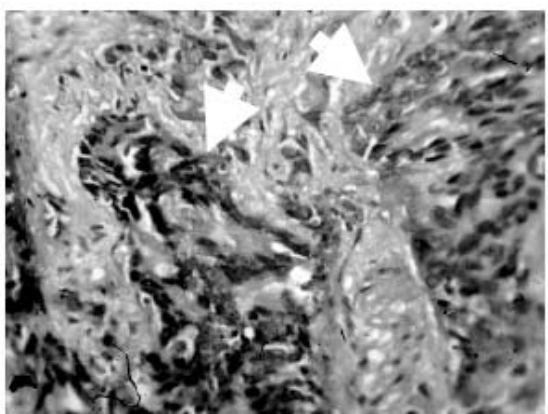

S20

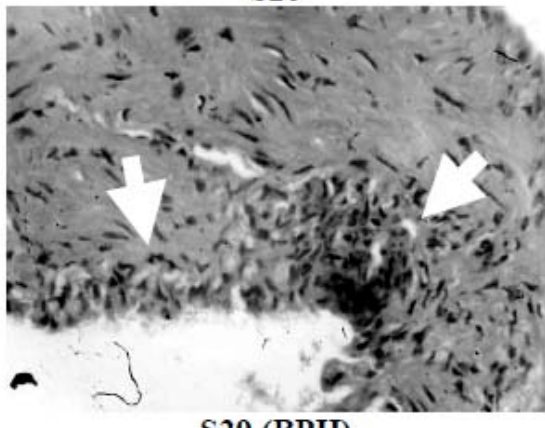

S29 (BPH)

Fig. (1). General morphology of prostate biopsies on $P C a$ and $B P H$ Diagnosed patients. Paraffin wax embedded sections were stained in Hematoxylin and Eosin following the methods of Zhang et al. (2012). It is important to note the tissue infiltration sites in PCa Biopsies (S09, S20 and S30) and increased cell proliferation in BPH epithelium (Magnification X400).

placed in preheated methenamine silver solution at $60^{\circ} \mathrm{C}$ for five minutes. Although it could be occasionally longer if the intensification had been carried out at room temperature. In this study, to further increase the clarity, Hematoxylin was removed from counterstained nuclei with $1 \%$ acid alcohol before the silver intensification was carried out. The composition of the stock solution was $0.125 \%$ silver nitrate in $1.5 \%$ hexamine. The solution was stored at $4{ }^{\circ} \mathrm{C}$. Prior to use, $2 \mathrm{ml}$ of $5 \%$ tetraborate was added to $50 \mathrm{ml}$ of the stock silver solution giving a $\mathrm{pH}$ of 8.0 , which was then filtered into a coupling jar and protected from sunlight.

\section{RESULTS AND DISCUSSION}

The expression of ECAD describes the progression of tumors to malignant cancers while that of $\mathrm{Ki}-67$ indicates the extent of aggressiveness of such cancers. The detached tumor cells shows ECAD immunonegativity compared with the surrounding tumor cells that showed ECAD immunopositivity (Fig. 2, PCa/S09). The extent of ECAD immunonegativity coincided with the extent of the cancer tissue invasion such that the lower the ECAD expression level, the more invasive the cancer cells. The Ki-67 however justifies the rate and extent of cell division within the $\mathrm{BPH}$ and PCa tissues; the BPH showed high ECAD expression, indicating that the epithelium is intact, although rate of cell proliferation and division is high as the BPH epithelium exhibits significant Ki-67 expression (Fig. 2, BPH/S29). Despite indication that cell proliferation is characteristic of both $\mathrm{BPH}$ and $\mathrm{PCa}$, the patterns of such proliferation differs to a large extent. Also, the epithelial integrity is maintained in BPH as against PCa where ECAD expression is reduced and cells are allowed to migrate from the epithelium to invade the surrounding glandular tissue (Fig. $3 \mathrm{BPH}$ and $\mathrm{PCa})$.

Scientific studies have showed that low level ECAD expression is an indication of onset malignancy in tumors. Although digital rectal examination captured both $\mathrm{PCa}$ and $\mathrm{BPH}$ as tumors, the neoplasm observed in $\mathrm{PCa}$ is not characteristic of BPH tissue and ECAD expression which is relatively high in the proliferating epithelium (Fig. 1). Both $\mathrm{PCa}$ and $\mathrm{BPH}$ can be described as a function of a dysregulated cell cycle control mechanism comprising of series of switch systems governed by cycling and cyclin dependent kinases (Cdks), which in turn controls specific protein and gene expression patterns. Like all other cell cycle control molecules, the kinases are important factors in 

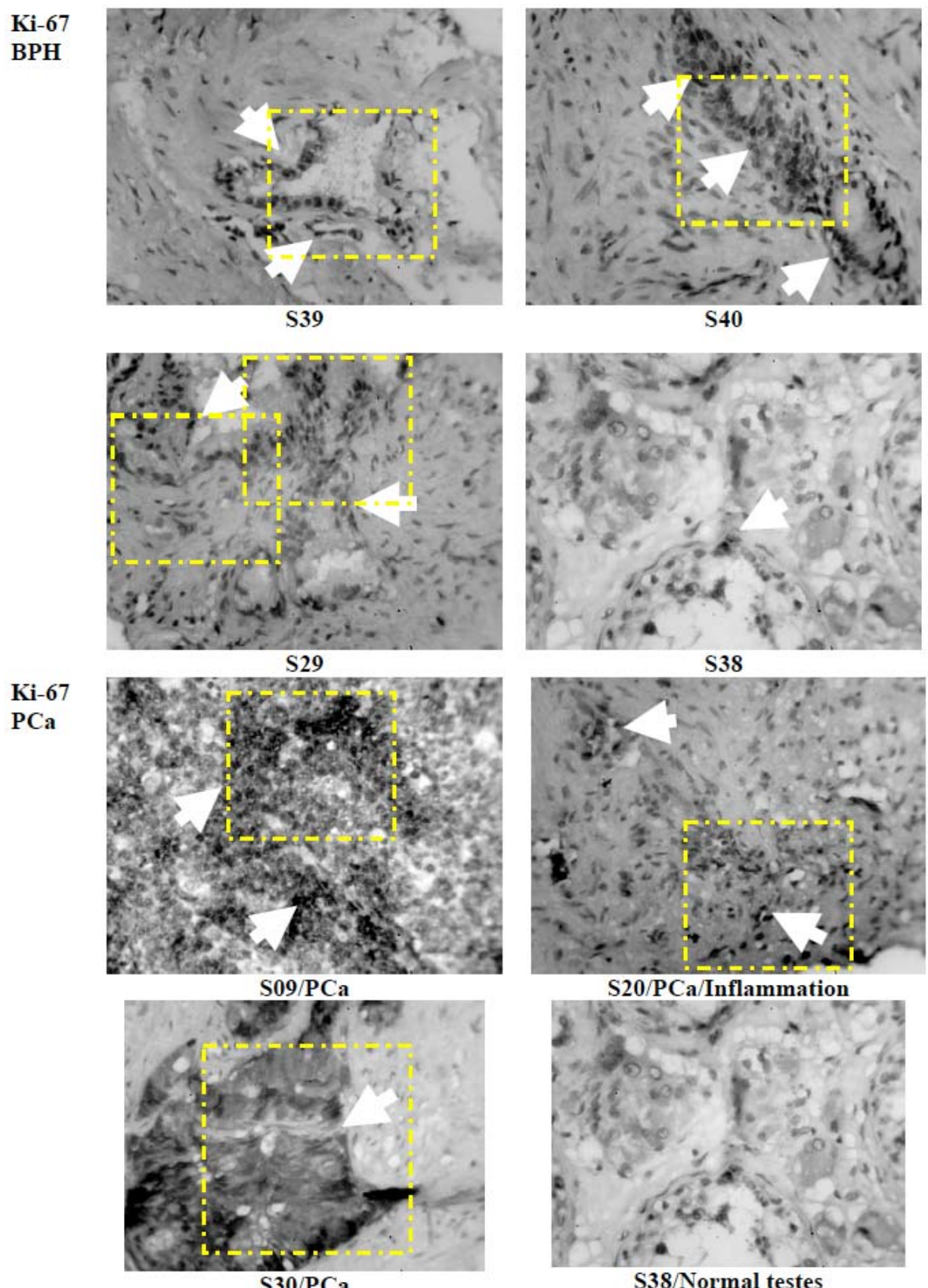

S38/Normal testes

Fig. (2). Immunohistochemical demonstration of $\mathrm{Ki}-67$ in the epithelium of BPH biopsies using anti Ki-67 MAb. It is important to note that immunopositivity in the tissues is restricted to the base of the epithelium and restricted to specific tissue sites (S29, S39 and S40). Over expression of Ki-67 was observed in the PCa biopsies, particularly in cell clumps located at random within tissues. High expression of Ki-67 in S09 and S30 shows rapid cell proliferation within the tumorgenic cell aggregations invading the glandular tissue of the prostate. (Magnification X400).

controlling the expression of ECAD and Ki-67. Certain cancers are not directly linked with the proteins but will rather affect the kinase system that controls the transcription level of the genes encoding for such proteins [14]. Other forms of cancer can result from DNA damage; this was mapped alongside with cell proliferation using $\mathrm{Ki}-67$ to immunohisto-chmeically differentiate these cells in $\mathrm{PCa}$ and $\mathrm{BPH}$ biopsies. The intensity of expression of these parameters was then compared between the groups to determine the extent of cell proliferation and malignancy between different PCa specimens and also between PCa and BPH specimens. Total Ki-67 expression levels corresponded with the extent of invasion of the tumor cells, being higher in the more invasive tumor cells that stained strongly with the anti-Ki-67 MAb and DAB (the polymer). ECAD expression levels also showed positive correlation with the extent of invasion. Although, certain cells that were found as isolated tumor masses were negative in immunohistochemistry for ECAD and only slightly positive for $\mathrm{Ki}-67$ showing that, though the cells were malignant, the tumor cells were less radical and invasive when compared with those with high levels of Ki-67 (Fig. 2 PCa/S09). The study also showed that high Ki-67 reactivity correlates with the histological grade of tumor observed in routine Hematoxylin and Eosin (Fig. 1). 


\section{ECAD PCa}

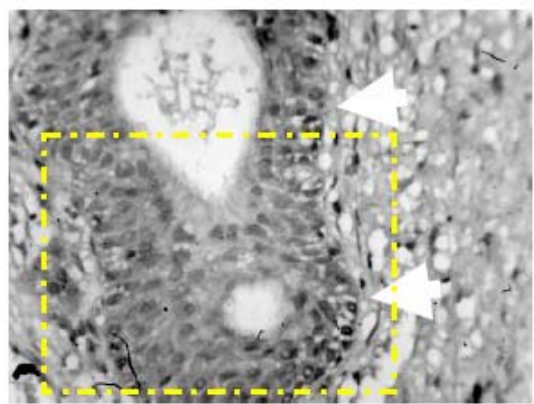

S09

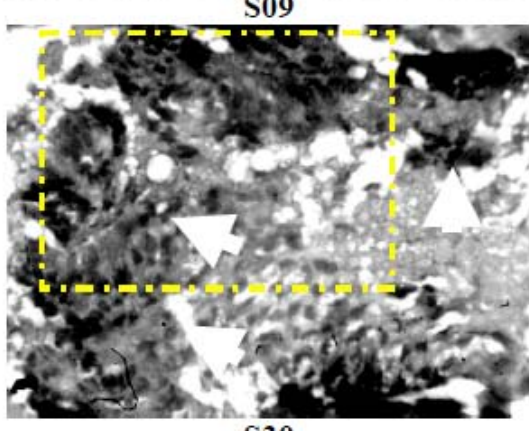

$$
\text { S30 }
$$

ECAD
BPH



S39

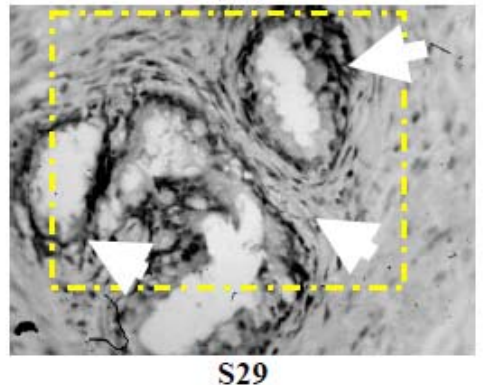

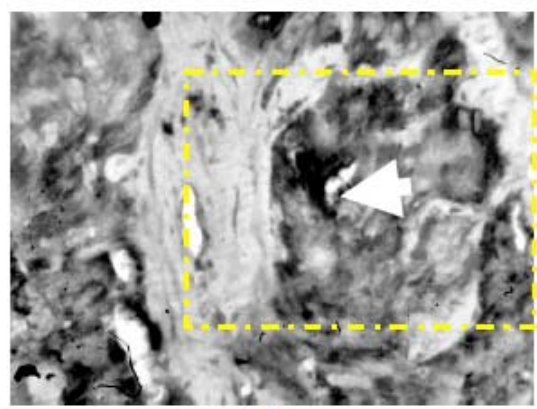

S20

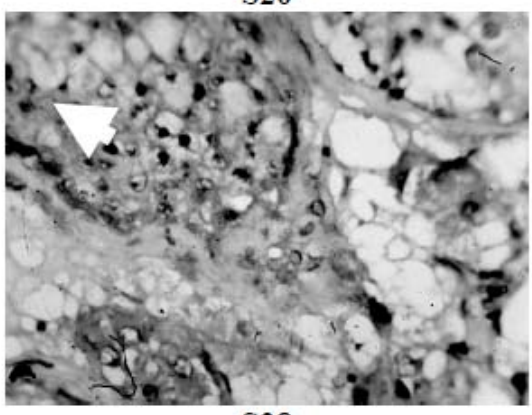

S38
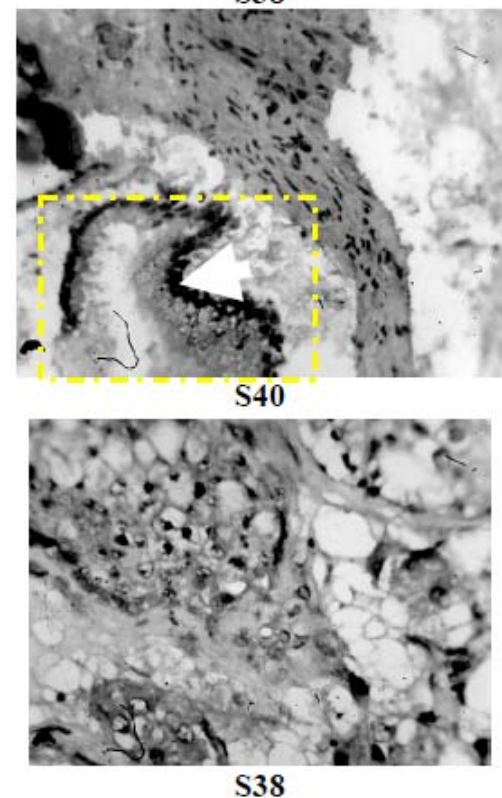

Fig. (3). ECAD positivity was restricted to the epithelium close to the origin of the $P C a$. Certain cells in the neoplasm show $E C A D$ negativity which depicts the loss of desmosomes or hemi-desmosomes. Such a tumor is most likely going to become malignant as it has detached from the tumor mass within the tissue. BPH: ECAD positivity was observed in the epithelial tissue of BPH biopsies, showing proliferation but no evidence of detachment. This is important as a control factor; although certain cells in $B P H$ biopsies were found to be CK7 positive. It is possible that inflamed cells resulting from stress factors in $B P H$ contributes to such immunopositivity rather than PCa. (Magnification $\mathrm{X} 400)$.

Most of the human cancers originate from the epithelium because they consist of cells that are characterized by a very high rate of proliferation [15]. The regular epithelium is organized by a series of intercellular junctional complexes usually the adherent junction and desmosomes that connects the cells to one another and to the cellular cytoskeleton of actin filaments and microtubules, such that the epithelium is organized into a framework of single cell layer barrier [16]. This cytoskeletal adhesion, however, is necessary to stabilize the epithelial structure. The cadherin molecules are important in maintaining the adherent junctions via calcium mediated cell-cell interaction [17]. One of the most predominant cadherin molecules is E-Cadherin, a transmembrane protein that creates ECAD interaction between 2 cells (calcium dependent) [1, 11]. It is logical to summarize that $C K 7$ can mark for $\mathrm{PCa}$ of glandular origin, thus important in characterizing neoplasm tissue mass at the interphase of epithelium and glandular tissue (Fig. 2 PCa). Finally, ECAD part positivity in glandular neoplastic tissue suggests tissue invasion by the cancer cells and the intact nature of others, while ECAD positivity in the epithelium of $B P H$ tissues supports cell proliferation with intact epithelial interaction rather than neoplasm and cell detachment (Fig. 3 PCa and Fig. 3 BPH). 
Different antibodies can directly label nuclear antigens expressed at the various stages of the cell cycle. The Ki-67 is a nuclear non histone protein that is related to the $S$ - and $M$ Phases and the associated G phases (G1 and G2) of the cell cycle; the protein has a molecular weight of $345 \mathrm{KDa}$. The use of this antibody as a proliferation marker is advantageous over flow cytometry as it combines both immunolabelling and immune-phenotyping, the turn over time is shorter than flow cytometry and it can assist clinicians in managing a lot of conditions. A major limitation of this technique in labeling ECAD and $\mathrm{Ki}-67$ is the requirement for antigen retrieval as certain antigens might have been destroyed during fixation and tissue processing. This study however provides deep insight into the labelling of Ki-67 and ECAD using immunohistochemistry, specifically the antigen retrieval in paraffin wax embedded sections to determine cell proliferation and epithelial integrity that can give a reasonable insight into the aggressive nature of tumor cells and the extent of metastasis observed in such cells.

\section{CONFLICT OF INTEREST}

The Authors hereby declare there is no conflict of interest associated with this study or any of the procedures and materials used for the purpose of the study.

\section{ACKNOWLEDGEMENTS}

Declared none.

\section{REFERENCES}

[1] Jiao L, Li Y, Shen D, et al. The prostate cancer-up-regulated mycassociated zinc-finger protein (MAZ) modulates proliferation and metastasis through reciprocal regulation of androgen receptor. Med Oncol 2013; 30(2): 570.

[2] Parkin J, Keeley FX Jr, Timoney AG. Laparoscopic lymph node sampling in locally advanced prostate cancer. BJU Int 2002; 89(1): 14-7.

[3] Berry SJ, Coffey DS, Walsh PC, Ewing LL. The development of human benign prostatic hyperplasia with age. J Urol 1984; 132(3): 474-9.
[4] Fisher G, Yang ZH, Kudahetti S. Prognostic value of Ki-67 for prostate cancer death in a conservatively managed cohort. Br J Cancer 2013; 108(2): 271-7.

[5] Hussain F, Freissmuth M, Volkel D, et al. Human AntiMacrophage Migration Inhibitory Factor (MIF) Antibodies Inhibit Growth of Human Prostate Cancer Cells In Vitro and In Vivo. Mol Cancer Ther 2013. [Epub ahead of print]

[6] Birchmeier W, Behrens J. Cadherin expression in carcinomas: role in the formation of cell junctions and the prevention of invasiveness. Biochim Biophys Acta 1994; 1198(1): 11-26.

[7] Mayer B, Johnson JP, Leitl F, et al. E-cadherin expression in primary and metastatic gastric cancer: down-regulation correlates with cellular dedifferentiation and glandular disintegration. Cancer Res 1993; 53(7): 1690-5.

[8] Ide T, Uchida K, Suzuki K, Kagawa Y, Nakayama H. Expression of cell adhesion molecules and doublecortin in canine anaplastic meningiomas. Vet Pathol 2011; 48(1): 292-301.

[9] Akat K, Bleck CK, Lee YM, Haselmann-Weiss U, Kartenbeck J. Characterization of a novel type of adherens junction in meningiomas and the derived cell line HBL-52. Cell Tissue Res 2008; 331(2): 401-12.

[10] Zee RY, Cheng S, Erlich HA, et al. Intercellular adhesion molecule 1 (ICAM1) Lys56Met and Gly241Arg gene variants, plasmasoluble ICAM1 concentrations, and risk of incident cardiovascular events in 23,014 initially healthy white women. Stroke 2007; 38(12): 3152-7.

[11] Monti S, Chapuy B, Takeyama K, et al. Integrative analysis reveals an outcome-associated and targetable pattern of p53 and cell cycle deregulation in diffuse large B cell lymphoma. Cancer Cell 2012; 22(3): 359-72.

[12] Pollack A, Cowen D, Troncoso P, et al. Molecular markers of outcome after radiotherapy in patients with prostate carcinoma: Ki67, bcl-2, bax, and bcl-x. Cancer 2003; 97(7): 1630-8

[13] Zhang ZZ, Gong YY, Shi YH, Zhang W, Qin XH, Wu XW. Valproate promotes survival of retinal ganglion cells in a rat model of optic nerve crush. Neuroscience 2012; 224: 282-93.

[14] Giton F, de la Taille A, Allory Y, et al. Estrone sulfate (E1S), a prognosis marker for tumor aggressiveness in prostate cancer (PCa). J Steroid Biochem Mol Biol 2008; 109(1-2): 158-67.

[15] Bougen NM, Amiry N, Yuan Y, et al. Trefoil factor 1 suppression of E-CADHERIN enhances prostate carcinoma cell invasiveness and metastasis. Cancer Lett 2013; 332(1): 19-29.

[16] De Barros MA, Panattoni MJF, Samoto VY, et al. Marsupial morphology of reproduction: South America opossum male model. Microsc Res Tech 2013; 76(4): 388-97.

[17] Tripathi P, Wang Y, Coussens M, et al. Activation of NFAT signaling establishes a tumorigenic microenvironment through cell autonomous and non-cell autonomous mechanisms. Oncogene 2013; doi: 10.1038/onc.2013.132 [Epub ahead of print] 